\title{
Modification of Rhodamine WT tracer tests procedure in activated sludge reactors
}

\author{
Marta Knap ${ }^{1, *}$, and Piotr Balbierz ${ }^{1}$ \\ ${ }^{1}$ Wroclaw University of Science and Technology, Faculty of Environmental Engineering, Wybrzeze \\ Wyspianskiego 27, 50-370 Wroclaw, Poland
}

\begin{abstract}
One of the tracers recommended for use in wastewater treatment plants and natural waters is Rhodamine WT, which is a fluorescent dye, allowing to work at low concentrations, but may be susceptible to sorption to activated sludge flocs and chemical quenching of fluorescence by dissolved water constituents. Additionally raw sewage may contain other natural materials or pollutants exhibiting limited fluorescent properties, which are responsible for background fluorescence interference. This paper presents the proposed modifications to the Rhodamine WT tracer tests procedure in activated sludge reactors, which allow to reduce problems with background fluorescence and tracer loss over time, developed on the basis of conducted laboratory and field experiments.
\end{abstract}

\section{Introduction}

Mixing and the intensity of mass exchange processes may have significant impact on the hydraulic efficiency of the activated sludge (AS) reactors and as a result the effectiveness of the wastewater treatment processes [1,2]. Determination of the hydrodynamic characteristics of AS reactors allows to detect mixing problems and is required during construction of wastewater treatment plant (WWTP) mathematical models. One of the methods used to determine the hydrodynamic models of AS reactors are tracer tests.

Due to the specific properties of the activated sludge, the raw sewage composition, dynamic working conditions and large reactors volumes, tracer experiments in AS reactors are time-consuming, cost- and labor-intensive. Morover, all stages of tracer tests including the choice of proper tracer, design and conduct of the experiment, as well as interpretation of results require more careful consideration. One of the tracers recommended for use in WWTPs, wetlands and natural waters is Rhodamine WT (RWT) [3].

\subsection{Rhodamine WT}

RWT is a fluorescent xanthene dye which is generally used as water tracer in various hydrodynamic studies (surface and ground water studies, rivers, estuaries, reservoirs and lakes studies, wetlands and wastewater treatment studies) for determining the hydraulic

\footnotetext{
*Corresponding author: marta.knap@pwr.edu.pl
} 
parameters and contaminant fate and transport. RWT is a useful tracers because it can be relatively easily detected with a field fluorometer at concentrations as low as $0.01 \mu \mathrm{g} / \mathrm{dm}^{3}$. Moreover RWT is non-toxic, non-carcinogenic and non-mutagenic even at relatively high concentrations of $2 \mathrm{mg} / \mathrm{dm}^{3}$ [4]. RWT has low sensitivity to photochemical decay and $\mathrm{pH}$-values, indicated by a constant maximal fluorescence in the $\mathrm{pH}$ range between 5 and $8[5]$.

RWT is commonly used as a conservative tracer in different hydrodynamic studies, including AS reactors hydrodynamic characterization. However, the literature on RWT reactivity, and in particular susceptibility to sorption to sediments, is somewhat conflicting. Some studies [6] consider the RWT as a conservative tracer, whereas other studies [7, 8] aimed at comparison of RWT and other conservative tracer such as $\mathrm{LiCl}$ and $\mathrm{Br}$ suitability in determination of hydraulic characteristics of constructed and natural wetlands, showed that RWT is less stable than $\mathrm{LiCl}$ and $\mathrm{Br}$.

Thorough laboratory research on the RWT sorption characteristics has shown that the extent of sorption is dependent on different factors such as sediment type, organic matter concentration, initial RWT concentration, $\mathrm{pH}$, ionic strength and the ionic composition of the solution [4, 9-12]. In addition, RWT is made up of two isomers [4, 9, 10] with different sorption properties and different emission spectra, which are equally distributed in RWT solution. Isomer 1 (para) is an almost conservative tracer, while isomer 2 (meta) is a nonconservative tracer due to its sorption to sediments $[4,9,10]$.

Studies have shown that another dye tracer Rhodamine B is not recommended for tracer tests due to susceptibility to sorption to sediments, but was used for specific surface area measurement of activated sludge [13].

However, there is no information about the RWT behaviour in the AS reactors. Therefore, the aim of this paper is to evaluate the suitability of RWT as a conservative tracer in hydrodynamic studies of AS reactors, based on its susceptibility to sorption to AS flocs and chemical quenching of fluorescence by dissolved water constituents assessed in laboratory batch tests using several concentrations of RWT and AS from two WWTPs in Poland.

\subsection{Tracer tests}

Tracer tests are conducted by introduction of a controlled input function (tracer addition) to the stream entering the AS reactor and subsequent measurements of tracer concentration at the outlet of the reactor as a function of time. Results of tracer tests provide approximate information about the mixing regime in reactors, based on the analyses of tracer response curves and determination of the hydraulic parameters, such as the hydraulic retention time (HRT), the number of tanks-in-series or the dispersion number, using numerical methods, e.g. the method of moments or simulation method.

Selection of the proper tracer for a paricular application has a key importance for tracer test success, as it determines further steps including tracer dose selection, samples preparation and detection method. To facilitate the selection of tracer the following criteria, that the ideal tracer should meet, were formulated [14-16]:

- physicochemical properties similar to the properties of wastewater (e.g. density),

- conservative (not absorbed on particles in wastewater and exposed reactor surfaces, nonreactive in any way in the reactor),

- non-toxic, non-carcinogenic and non-mutagenic at used concentrations,

- easy and inexpensive detection at low concentrations,

- low price and availability on the market. 
In practice, selection of the tracer that simultaneously meets all of the above criteria is nearly impossible. Therefore, the choice of tracer is usually a compromise between its positive and negative properties.

Although RWT is susceptible to some extent to sorption to sediments and chemical quenching of fluorescence by dissolved water constituents, it provides easy detection with a field fluorometer at low concentrations, relatively low prices, and has no negative influence on living organisms. The general procedure for performing tracer tests using RWT is presented elsewhere [3].

Taking into account the numerous problems and doubts involved in conducting tracer tests in AS reactors, the authors conducted additional experiments and suggested modification the general tracer test procedure to adapt it to the specificity conditions of AS reactors in WWTPs.

\section{Material and methods}

A series of laboratory and field tests were conducted to determine background fluorescence interference and the loss of tracer due to sorption to AS flocs in real WWTPs conditions.

Background fluorescence and RWT concentrations were analysed in prefiltered samples using filter fluorometer (Quantech Base) at an excitation wavelength of $550 \mathrm{~nm}$ and an emission wavelength of $580 \mathrm{~nm}$.

The 20\% RWT aqueous solution (Keystone) was used to prepare RWT calibration curves and in all laboratory batch tests.

\subsection{Background fluorescence determination}

Background fluorescence in wastewater and its variability over time was determined in field studies conducted at two Leszno and Wroclaw WWTPs (Poland).

The test at WWTP in Leszno was carried out for 50 hours. Samples were taken at one sampling point, the inlet to the anaerobic reactor (AN), every $30 \mathrm{~min}$ for the first $2 \mathrm{~h}$ and every $2 \mathrm{~h}$ for another 48 hours. All samples were immediately filtered through medium filter paper and subsequently analysed for fluorescence (at RWT parameters).

The test at WWTP in Wroclaw was carried out for $7 \mathrm{~h}$ (the typical tracer test time). Samples were collected at three sampling points, the inlet to the oxidation reactor (OX), the outlet of the OX and the internal recirculation stream every $1 \mathrm{~h}$ for 7 hours. All samples were immediately filtered through medium filter paper and subsequently analysed for fluorescence.

\subsection{Laboratory sorption studies}

RWT susceptibility to sorption to AS flocs and the tracer loss over time was determined in laboratory batch tests conducted on AS samples collected from Leszno and Wroclaw WWTPs.

Three sorption experiments were conducted on AS samples collected from the bioreactor of Wroclaw WWTP. In the first and second tests, three laboratory reactors equipped with a mechanical stirrer were filled with $3.0 \mathrm{dm}^{3}$ of $\mathrm{AS}$, then appropriate quantities of RWT ( $20 \%$ aqueous solution) were added so that the resultant concentrations were 100,50 and $10 \mu \mathrm{g} \mathrm{RWT} / \mathrm{dm}^{3}$. Samples were collected every $30 \mathrm{~min}$. for $10 \mathrm{~h}$ (first test), and every $30 \mathrm{~min}$. for the first $4 \mathrm{~h}$ and every $1 \mathrm{~h}$ for another $6 \mathrm{~h}$ (second test). All samples were immediately filtered through medium filter papers and subsequently analysed for fluorescence. 
In the third sorption experiment the three laboratory reactors were filled with $3.0 \mathrm{dm}^{3}$ of AS at different concentrations. Sludge concentrations in reactors R1 and R3 were respectively approximately two times lower (dilution with tap water) and higher (thickened by sedimentation) compared to reactor R2.

Then appropriate quantities of RWT (20\% aqueous solution) were added so that the resultant concentration was $50 \mu \mathrm{g} \mathrm{RWT} / \mathrm{dm}^{3}$. Samples were collected every $30 \mathrm{~min}$. for the first $2 \mathrm{~h}$ and every $1 \mathrm{~h}$ for another $6 \mathrm{~h}$. All samples were immediately filtered through medium filter papers and subsequently analysed for fluorescence.

The fourth sorption experiment was conducted on AS sample collected from the bioreactor of Leszno WWTP. Three laboratory reactors were filled in $3.0 \mathrm{dm}^{3}$ of AS, then appropriate quantities of RWT (20\% aqueous solution) were added so that the resultant concentrations were 100,50 and $10 \mu \mathrm{g}$ RWT/ $\mathrm{dm}^{3}$. Samples were collected every $30 \mathrm{~min}$. for $10 \mathrm{~h}$. All samples were immediately filtered through medium filter papers and subsequently analysed for fluorescence.

\section{Results and discussion}

\subsection{Background fluorescence determination results}

Background fluorescence in wastewater and its variability over time determined in field studies conducted at Leszno and Wroclaw WWTPs is shown at Figure 1. The emission values read in the samples were converted to the corresponding RWT concentrations (based on the RWT calibration curve).

The average background fluorescence in samples taken at Leszno WWTP corresponds to RWT concentration of $0.18 \mu \mathrm{g} / \mathrm{dm}^{3}$ with the standard deviation of $0.014 \mu \mathrm{g} / \mathrm{dm}^{3}$. The average background fluorescence in samples taken at Wroclaw WWTP at the inlet and outlet of OX reactor corresponds to RWT concentration of $0.18 \mu \mathrm{g} / \mathrm{dm}^{3}$, whereas in samples taken from internal recirculation to RWT concentration of $0.26 \mu \mathrm{g} / \mathrm{dm}^{3}$, with the standard deviation of $0.018,0.012$ and $0.010 \mu \mathrm{g} / \mathrm{dm}^{3}$, respectively.

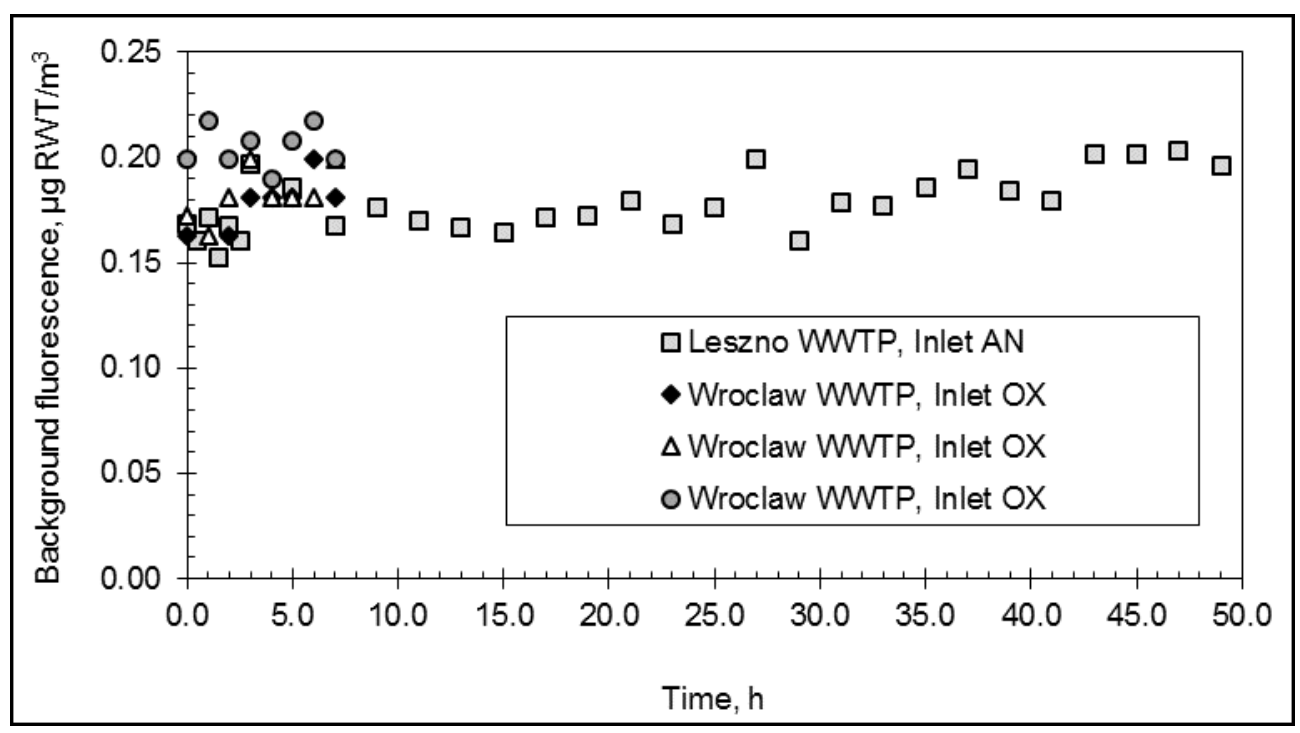

Fig. 1. Background fluorescence interference at Leszno and Wroclaw WWTPs. 
The results suggest that the average background fluorescence should be determined and taken into account during tracer test analysis, whereas its temporal variation usually may be neglected. The RWT fluorescence should be adjusted by manually subtracting the background component or automatically resetting the background fluorescence to zero (if the fluorometer has such a function). Due to the varied composition of municipal wastewater, the authors suggest that the background fluorescence should be determined in field experiments for each WWTP, directly before or if possible simultaneously to the actual tracer tests.

\subsection{Sorption studies results}

The relative RWT loss over time, due to sorption to sediments and/or chemical quenching of fluorescence by dissolved water constituents (effects which are indistinguishable in this experiment), recorded during the first test was shown in Figure 2. In all the experiments, the effect of the initial RWT concentration on the degree of RWT loss was negligible, therefore, only the results of one experiment at three concentration were presented in details (Fig. 2) and in further considerations the average values of three concentrations were used.

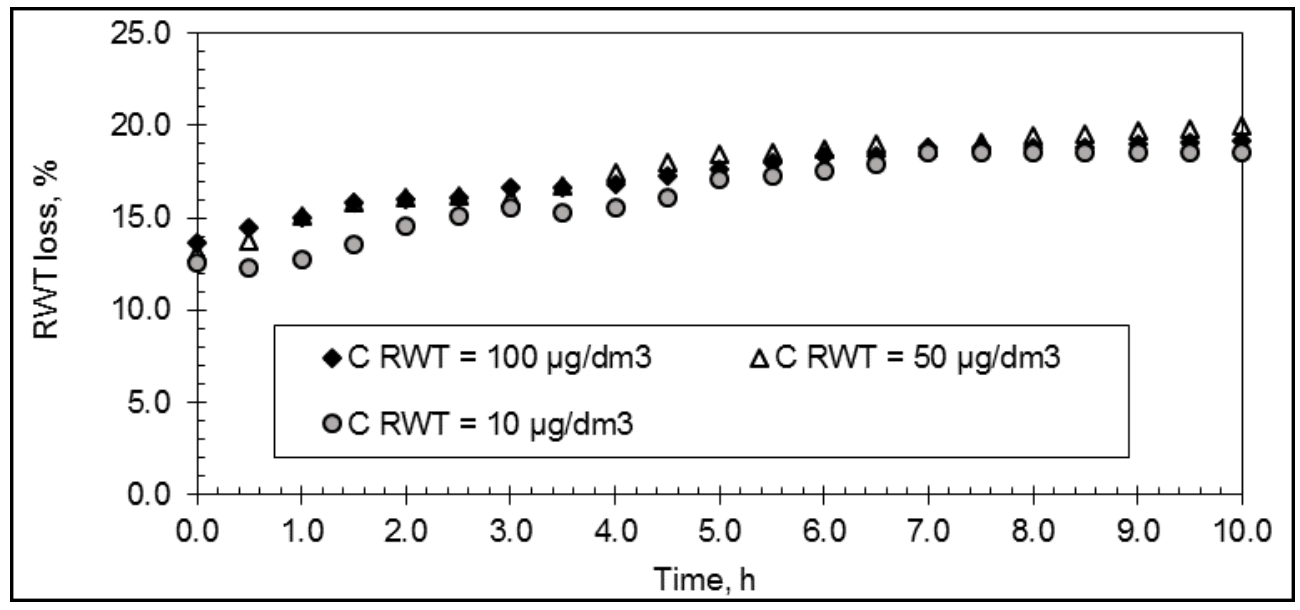

Fig. 2. RWT concentration loss over time in test I (MLSS $=3.20 \mathrm{~g} \mathrm{TSS} / \mathrm{dm}^{3}$ and $2.10 \mathrm{~g} \mathrm{VSS} / \mathrm{dm}^{3}$ ).

The average relative RWT losses over time, due to sorption to sediments and/or chemical quenching of fluorescence by dissolved water constituents, recorded during the first, second and fourth test were shown in Figure 3. The degree of RWT loss in these tests was significantly different, despite relatively similar sludge concentrations, and amounted to $17.1 \%, 21.6 \%$, and $10.6 \%$ in the first, second and fourth test, respectively. These results suggest that it is not the sludge concentration, but mainly the composition of activated sludge and wastewater that has a significant effect on RWT loss. Also the initial RWT concentration in the samples that were taken immediately after RWT addition was respectively $12.5 \%, 17.1 \%$ and $9.5 \%$ lower than theoretical concentration, which suggest that the initial loss of RWT is instantaneous.

The relative RWT loss over time, due to sorption to sediments and/or chemical quenching of fluorescence by dissolved water constituents, recorded during the third test at various sludge concentrations was shown in Figure 4. The RWT loss over time at relatively low sludge concentrations in reactors R1 (MLSS $=1.75 \mathrm{~g} \mathrm{TSS} / \mathrm{dm}^{3}$ and $1.27 \mathrm{~g} \mathrm{VSS} / \mathrm{dm}^{3}$ ) and R2 (MLSS $=3.40 \mathrm{~g} \mathrm{TSS} / \mathrm{dm}^{3}$ and $2.47 \mathrm{~g} \mathrm{VSS} / \mathrm{dm}^{3}$ ) amounted to $15.2 \%$ and $16.9 \%$, respectively, and did not differ significantly. Whereas, a significantly higher tracer loss of 
28.7\% was observed in reactor R3 filled with sludge at the high concentration $\left(\mathrm{MLSS}=6.908 \mathrm{~g} \mathrm{TSS} / \mathrm{dm}^{3}\right.$ and $\left.4.952 \mathrm{~g} \mathrm{VSS} / \mathrm{dm}^{3}\right)$.

The obtained results suggests that the RWT loss is independent of the initial tracer concentration, but is strongly dependent on the activated sludge and wastewater composition, and to a limited extent on sludge concentration (increase in the amount of adsorbed tracer occurs only at sufficiently high organic matter concentration). In order to model the variability of the RWT loss over time, the linear equations were matched to the results of the experiments (Fig. 3 and 4). The RWT loss over time is linear at specified intervals.

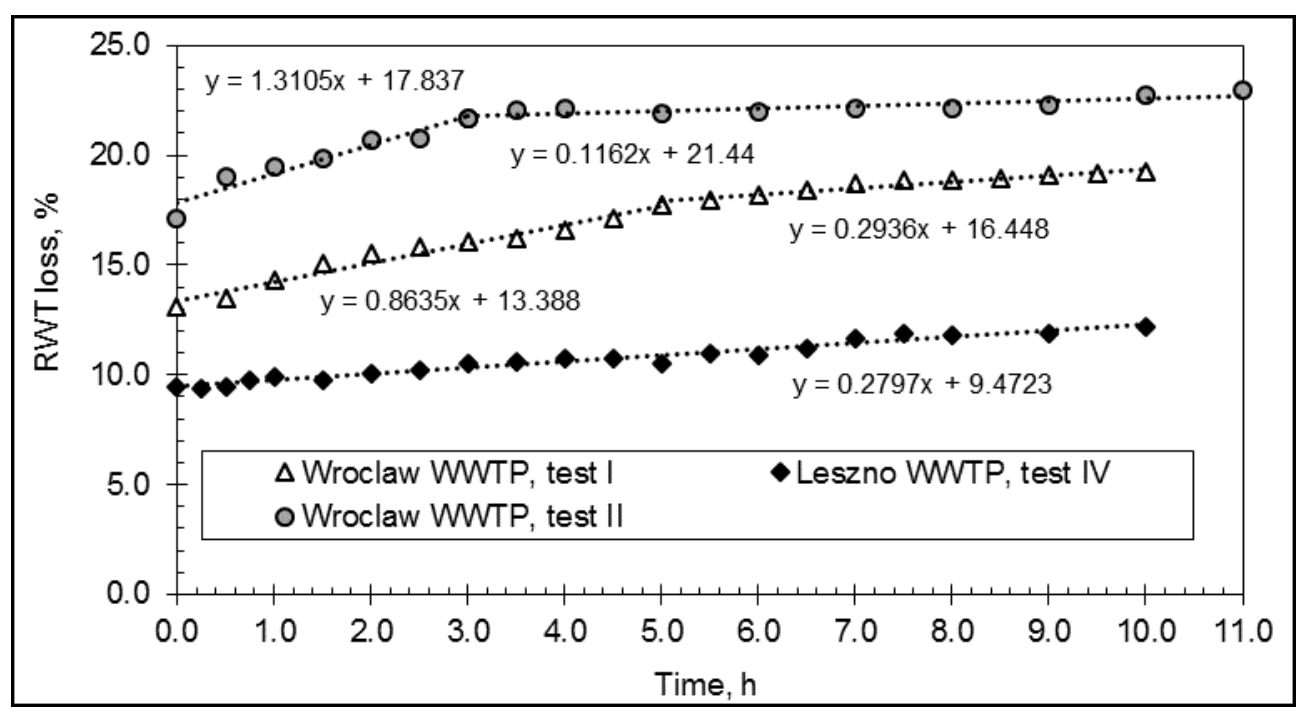

Fig. 3. Average RWT loss over time in test I (MLSS $=3.20 \mathrm{~g} \mathrm{TSS} / \mathrm{dm}^{3}$ and $2.10 \mathrm{~g} \mathrm{VSS} / \mathrm{dm}^{3}$ ), test II $\left(\right.$ MLSS $=3.52 \mathrm{~g} \mathrm{TSS} / \mathrm{dm}^{3}$ and $2.20 \mathrm{~g} \mathrm{VSS} / \mathrm{dm}^{3}$ ), test IV $\left(\right.$ MLSS $=3.8 \mathrm{~g} \mathrm{TSS} / \mathrm{dm}^{3}$ and $2.53 \mathrm{~g} \mathrm{VSS} / \mathrm{dm}^{3}$ ).

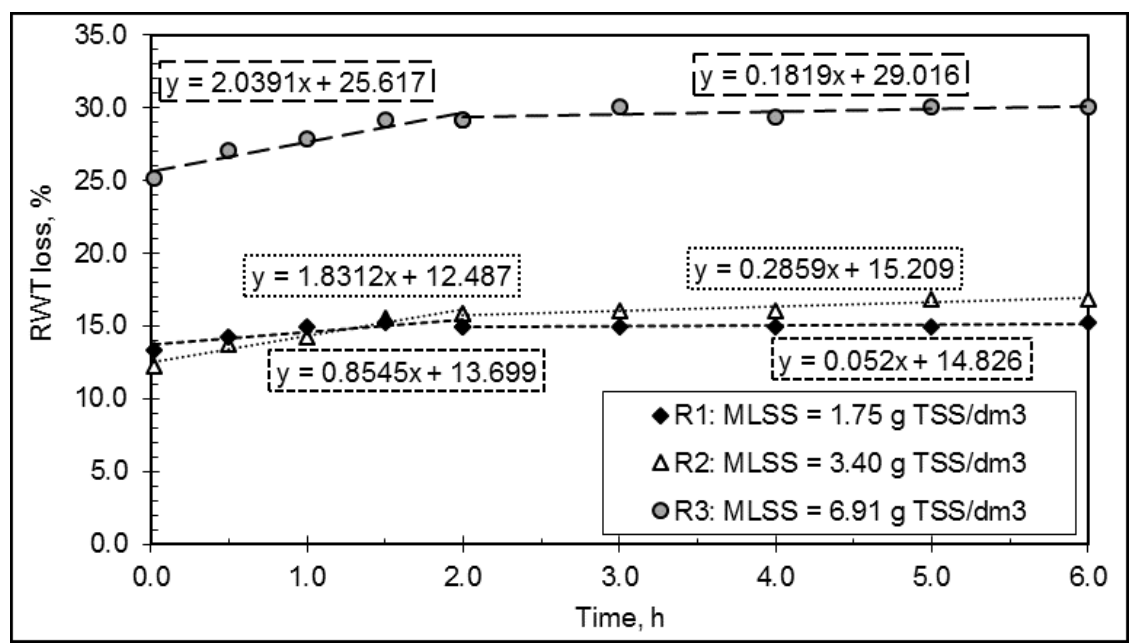

Fig. 4. RWT loss over time in reactor I (MLSS $=1.75 \mathrm{~g} \mathrm{TSS} / \mathrm{dm}^{3}$ and $1.27 \mathrm{~g} \mathrm{VSS} / \mathrm{dm}^{3}$ ), reactor II (MLSS $=3.40 \mathrm{~g} \mathrm{TSS} / \mathrm{dm}^{3}$ and $2.47 \mathrm{~g} \mathrm{VSS} / \mathrm{dm}^{3}$ ), reactor III $\left(\mathrm{MLSS}=6.91 \mathrm{~g} \mathrm{TSS} / \mathrm{dm}^{3}\right.$ and $\left.4.95 \mathrm{~g} \mathrm{VSS} / \mathrm{dm}^{3}\right)$. 
Due to the varied composition of municipal wastewater and activated sludge, the authors suggest that RWT susceptibility to sorption on sediments over time should be verified in laboratory tests for each WWTP, directly before or if possible simultaneously to the actual tracer tests.

\section{Conclusions}

Although RWT is a non-conservative tracer, and may be susceptible to some extent to sorption to sediments and chemical quenching of fluorescence by dissolved water constituents, but it provides easy detection with field fluorometers at low concentrations, relatively low costs, and has no negative influence on living organisms, therefore it can be successfully used for tracer tests in AS reactors

The results of conducted studies suggest that RWT is susceptible to sorption to AS flocs and/or to chemical quenching of fluorescence by dissolved water constituents in a mixture of activated sludge and wastewater. The RWT loss is independent of the initial tracer concentration, but is strongly dependent on the activated sludge and wastewater composition, and to a limited extent depends on sludge concentration (increase in the amount of adsorbed tracer occurs only at sufficiently high organic matter concentration). The RWT loss over time is linear at specified intervals.

Consequently, the use of RWT as a conservative tracer may by the reason for poor mass recovery and errors in the tracer tests interpretation. Some researchers claim that the poor tracer mass recovery and the non-conservative character of RWT does not adversely affect the quality of the tracer test results, especially if the method of moments is used for interpretation $[6,8,17]$. However, if the results interpretation is based on a simulation method that involves fitting the model curve directly to the tracer test results these discrepancies may be important.

To facilitate the interpretation of RWT tracer test in AS reactors and to eliminate the interferences caused by background fluorescence as well as adsorption to AS flocs and/or chemical quenching of fluorescence by dissolved water constituents, the following modification of the standard tracer tests procedure are suggested:

- Determination of background fluorescence based on the results of field experiments. The background fluorescence should be properly accounted for when analysing tracer tests samples. Knowledge about background fluorescence is also necessary to correctly estimate the required tracer mass. Ideally, background fluorescence should be an insignificant fraction of the RWT concentration in the tracer test.

- Determination of RWT loss over time due to adsorption to AS flocs and/or chemical quenching of fluorescence by dissolved water constituents based on the results of a batch laboratory experiment. The RWT loss over time should be properly modelled and taken into account in the tracer test interpretation.

\section{References}

1. Metcalf \& Eddy, Wastewater Engineering: Treatment and Resource Recovery (G. Tchobanoglous, F.L. Burton, H.D. Stensel, ed., McGraw-Hill Education, 2014)

2. Y. Le Moullec, O. Potier, C. Gentric, J.P. Leclerc, Chem. Eng. Sci. 63, 2436-2449 (2008)

3. USGS Fluorometric procedures for dye tracing, United States Government Printing Office, Washington (1986)

4. D. Vasudevan, R.L. Fimmen, A.B. Francisco, Environ. Sci. Technol. 35, 4089-4096 (2001) 
5. W. Käss, Tracing technique in geohydrology (A.A Balkema, Rotterdam, Brookfield, 1998)

6. L. Pang, M. Close, M. Noonan, Ground Water 36, 1, 112-122 (1998)

7. A.Y.-C. Lin, J.-F. Debroux, J.A. Cunningham, M. Reinhard, Ecol. Eng. 20, 75-88 (2003)

8. F.E. Dierberg, T.A. DeBusk, Wetlands 25, 8-25 (2005)

9. B. J. Shiau, D. A. Sabatini, J. H. Harwell, Ground Water 31, 913-920 (1993)

10. D.J. Sutton, Z.J. Kabala, A. B. Francisco, D. Vasudevan, Water Resour. Res. 37, 1641-1656 (2001)

11. T. Kasnavia, D. A. Sabatini, Ground Water 37, 376-381 (1999)

12. D.A. Sabatini, T.A. Austin, Ground Water 29, 341-349 (1991)

13. B.L. Sorensen, R.J. Wakeman, Water Res. 30, 115-121 (1996)

14. J. Makinia, Mathematical modelling and computer simulation of activated sludge systems (London, New York, IWA Pub, 2010)

15. E. Iller, Badania znacznikowe w inżynierii procesowej (WNT, Warszawa, 1992)

16. Metcalf \& Eddy, Wastewater Engineering: Treatment and Reuse (G. Tchobanoglous, F.L. Burton, H.D. Stensel, ed., McGraw-Hill Education, 2004)

17. D. Giraldi, M. de'Michieli Vitturi, M. Zaramella, A Marion, R Iannelli, Ecol. Eng. 35, 265-273 (2009) 